\title{
NGTS-5b: a highly inflated planet offering insights into the sub-Jovian desert ${ }^{\star}$
}

Philipp Eigmüller ${ }^{1,2}$, Alexander Chaushev ${ }^{2}$, Edward Gillen ${ }^{7,11}$, Alexis Smith ${ }^{1}$, Louise D. Nielsen ${ }^{8}$, Oliver Turner ${ }^{8}$, Szilard Csizmadia ${ }^{1}$, Barry Smalley ${ }^{15}$, Daniel Bayliss ${ }^{4,5}$, Claudia Belardi ${ }^{6}$, François Bouchy ${ }^{8}$, Matthew R. Burleigh ${ }^{6}$, Juan Cabrera ${ }^{1}$, Sarah L. Casewell ${ }^{6}$, Bruno Chazelas ${ }^{8}$, Benjamin F. Cooke ${ }^{4,5}$, Anders Erikson ${ }^{1}$, Boris T. Gänsicke ${ }^{4,5}$, Maximilian N. Günther ${ }^{7}$, Michael R. Goad ${ }^{6}$, Andrew Grange ${ }^{6}$, James A. G. Jackman ${ }^{4,5}$, James S. Jenkins ${ }^{9,10}$, James McCormac ${ }^{4,5}$, Maximiliano Moyano ${ }^{12}$, Don Pollacco ${ }^{4,5}$, Katja Poppenhaeger ${ }^{\star \star}, 13,14$, Didier Queloz ${ }^{7}$, Liam Raynard ${ }^{6}$, Heike Rauer ${ }^{1,2,3}$, Stéphane Udry ${ }^{8}$, Simon. R. Walker ${ }^{4,5}$, Christopher A. Watson ${ }^{\star \star}$, Richard G. West ${ }^{4,5}$, and Peter J. Wheatley ${ }^{4,5}$

\footnotetext{
${ }^{1}$ Institute of Planetary Research, German Aerospace Center, Rutherfordstrasse 2, 12489 Berlin, Germany e-mail: philipp.eigmueller@dlr.de

${ }^{2}$ Center for Astronomy and Astrophysics, TU Berlin, Hardenbergstr. 36, 10623 Berlin, Germany

${ }^{3}$ Institute of Geological Sciences, FU Berlin, Malteserstr. 74-100, 12249 Berlin, Germany

${ }^{4}$ Centre for Exoplanets and Habitability, University of Warwick, Gibbet Hill Road, Coventry CV4 7AL, UK

${ }^{5}$ Department of Physics, University of Warwick, Gibbet Hill Road, Coventry CV4 7AL, UK

${ }^{6}$ Department of Physics and Astronomy, Leicester Institute of Space and Earth Observation, University of Leicester, LE1 7RH, UK

${ }^{7}$ Astrophysics Group, Cavendish Laboratory, J.J. Thomson Avenue, Cambridge CB3 0HE, UK

${ }^{8}$ Observatoire de Genève, Université de Genève, 51 Ch. des Maillettes, 1290 Sauverny, Switzerland

${ }_{9}^{9}$ Departamento de Astronomia, Universidad de Chile, Casilla 36-D, Santiago, Chile

${ }^{10}$ Centro de Astrofísica y Tecnologías Afines (CATA), Casilla 36-D, Santiago, Chile

${ }_{11}$ Astrophysics Research Centre, School of Mathematics and Physics, Queen's University Belfast, BT7 1NN Belfast, UK

12 Instituto de Astronomía, Universidad Católica del Norte, Angamos 0610, 1270709 Antofagasta, Chile

${ }^{13}$ Leibniz Institute for Astrophysics Potsdam (AIP), An der Sternwarte 16, 14482 Potsdam, Germany

${ }^{14}$ Institute for Physics and Astronomy, University of Potsdam, Campus Golm, Karl-Liebknecht-Str. 24/25, 14476 Potsdam, Germany

15 Astrophysics Group, Keele University, Staffordshire ST5 5BG, UK
}

Received 5 February 2019 / Accepted 25 April 2019

\begin{abstract}
Context. Planetary population analysis gives us insight into formation and evolution processes. For short-period planets, the subJovian desert has been discussed in recent years with regard to the planet population in the mass/period and radius/period parameter space without taking stellar parameters into account. The Next Generation Transit Survey (NGTS) is optimised for detecting planets in this regime, which allows for further analysis of the sub-Jovian desert.

Aims. With high-precision photometric surveys (e.g. with NGTS and TESS), which aim to detect short period planets especially around M/K-type host stars, stellar parameters need to be accounted for when empirical data are compared to model predictions. Presenting a newly discovered planet at the boundary of the sub-Jovian desert, we analyse its bulk properties and use it to show the properties of exoplanets that border the sub-Jovian desert.

Methods. Using NGTS light curve and spectroscopic follow-up observations, we confirm the planetary nature of planet NGTS-5b and determine its mass. Using exoplanet archives, we set the planet in context with other discoveries.

Results. NGTS-5b is a short-period planet with an orbital period of $3.3569866 \pm 0.0000026$ days. With a mass of $0.229 \pm 0.037 M_{\text {Jup }}$ and a radius of $1.136 \pm 0.023 R_{\text {Jup }}$, it is highly inflated. Its mass places it at the upper boundary of the sub-Jovian desert. Because the host is a K2 dwarf, we need to account for the stellar parameters when NGTS-5b is analysed with regard to planet populations.

Conclusions. With red-sensitive surveys (e.g. with NGTS and TESS), we expect many more planets around late-type stars to be detected. An empirical analysis of the sub-Jovian desert should therefore take stellar parameters into account.
\end{abstract}

Key words. planets and satellites: detection - planets and satellites: gaseous planets

\footnotetext{
* Based on observations made with CORALIE echelle spectrograph mounted on the $1.2 \mathrm{~m}$ Swiss telescope and the HARPS spectrograph on the ESO $3.6 \mathrm{~m}$ telescope, both at La Silla observatory (ESO, Chile). HARPS programme IDs are 098.C-0820(A), 099.C-0303(A), 0100.C-0474(A), and 0101.C-0623(A).

$\star \star$ Winton Fellow.
} 


\section{Introduction}

Thousands of extrasolar planets have been discovered by now, several hundred of which are well characterised by their bulk properties. Although these exoplanets are distributed over a wide parameter space, it is evident that we have reached a time when we can perform detailed population analysis and set our discoveries in context of planet formation and planetary system evolution theories. One of the discovered groups are the very hot short-period planets with orbital periods of only a few days. Population analysis of these short-period planets that were discovered with ground-based and space-borne surveys suggests a significant dearth of hot-Neptunian planets. This so called "sub-Jovian desert" cannot be explained by observational bias but seems to be related to the physical processes in the evolution of these hot planets. A detailed empirical study of the location of the sub-Jovian desert has been carried out by Mazeh et al. (2016). They analysed the population for these short-period planets in the mass-period and radius-period parameter space. Although the absence of short-period planets for a certain mass or radius has been proven, it is not clear which physical processes are responsible.

Possible effects include photo-evaporation (Lundkvist et al. 2016), which strips the planet of its atmosphere and reduces it in size and mass. This could explain especially the lower boundary of the sub-Jovian desert in relation to planetary mass as well as in regard to radius. Photo-evaporation is related to the incidence of UV/X-ray photons that heat the upper atmosphere of the planet. Another observed effect is the inflation of hot Jupiters, a phenomenon that is related to the equilibrium temperature of the planet. It is not completely understood what causes this inflation. Possible explanations amongst others include ohmic heating (Laughlin et al. 2011), tidal dissipation (Bodenheimer et al. 2001), kinetic heating (Guillot \& Showman 2002), and enhanced atmospheric opacities (Burrows et al. 2007). The recent study of inflated radii of hot-Jupiter planets by Sestovic et al. (2018) analysed the relationship of radius to stellar irradiation and mass. Radius inflation might play an important role in the location and shape of the upper boundary of the sub-Jovian desert in the radius/period parameter space. Independent of these effects, Matsakos \& Königl (2016) showed that high-eccentricity migration alone can explain the observed population. A recent study by Owen \& Lai (2018) combined the effects of photoevaporation, planetary inflation, and high-eccentricity migration to explain the observed planet distribution in the mass/period and radius/period parameter space.

Meanwhile, the number of detected short-period planets increases with highly sensitive ongoing and new surveys (e.g. K2; Howell et al. 2014, Next Generation Transit Survey, NGTS; Wheatley et al. 2018, TESS; Ricker et al. 2010). Some of these discoveries lie at the very edge or even within the sub-Jovian desert (e.g. Eigmüller et al. 2017; West et al. 2019). With the ongoing high-sensitivity surveys that target short-period planet discoveries, we expect to find many more planets in this regime. As these new surveys are optimised towards redder stars, most of these planets will likely be detected around smaller stars. This will allow us to analyse the sub-Jovian desert not only as a homogeneous sample, but will also permit us to investigate the planet distribution as a function of different stellar parameters such as mass, luminosity, temperature, and metallicity, presumably allowing tests of prediction by different explanations for the desert.

Presenting a short-period planet around a K-type star that was newly discovered by NGTS, we discuss the sub-Jovian desert and how the stellar type could be accounted for in future discussions of the dearth of short-period sub-Jovian planets. First we concentrate on the newly discovered planet NGTS-5b. In Sect. 2 the observations are described, followed by a description of the analysis (Sect. 3) and the resulting parameters for NGTS-5b (Sect. 4). In Sect. 5 we discuss NGTS-5b and place it in context of other planets. We also discuss the effect of the host star on the sub-Jovian desert. Finally, we summarise our results in Sect. 6.

\section{Observations}

\subsection{NGTS observation}

NGTS (Chazelas et al. 2012; Wheatley et al. 2018) is a dedicated ground-based transit survey that photometrically monitors millions of stars between 10th and 15th magnitude in $V$ mag. It consists of 12 independent telescopes each with a field of view of 8 square degrees. The red-sensitive CCD cameras allow us to detect signals of $1 \mathrm{mmag}$ for stars brighter than 13th magnitude. The main goal of the survey is to detect Neptune-sized planets around K-type stars. To allow us to detect planets around lowmass stars, the photometric bandwidth of the instrument was selected to be $580-920 \mathrm{~nm}$. A search for planets around lowmass stars offers two advantages. On the one hand, the number of confirmed planets around M- and K-type stars is still quite low and is the limiting factor of statistical analyses of planetary populations around host stars of different stellar types. On the other hand, a planet around a small star produces a much stronger transit and radial velocity signal than a planet around a G-type star.

Regular observations of NGTS started in April of 2016, and the first discoveries (e.g. Bayliss et al. 2018; Raynard et al. 2018; Günther et al. 2018; West et al. 2019; Jackman et al. 2018) have recently been reported. The first NGTS data release is publicly available in the ESO archive.

For the transit survey, each telescope is pointed independently at a single field. NGTS-5 has been observed from January until September 2016 on 112 observing nights. All together over 85000 observations, each with 10s exposure time, have been gathered by NGTS.

The NGTS data were processed using the NGTS pipeline as described in Wheatley et al. (2018). First a standard data reduction, including a bias subtraction and a flat field correction, was applied to all frames. To generate the light curve, aperture photometry based on the CASUtools (Irwin et al. 2004) software package was performed. To remove the most dominant systematic effects, the SysRem algorithm (Tamuz et al. 2005) was used. Finally, using our own implementation of the BLS algorithm (Kovács et al. 2002), NGTS-5b was detected. The phase-folded light curve is shown in Fig. 1. When we vetted the candidate, we found no signs that would indicate an eclipsing binary system. We also used the centroid vetting procedure (Günther et al. 2017) to rule out contamination from background objects. After passing all vetting steps, we followed-up NGTS-5b to confirm the planetary nature of the system and measure the planetary parameters.

\subsection{Photometric follow-up}

To constrain the ephemeris of the planet, candidate photometric follow-up observations at the South African Astronomical Observatory (SAAO) and the La Silla Observatory (Chile) were carried out. The observations are shown in Fig. 2. 


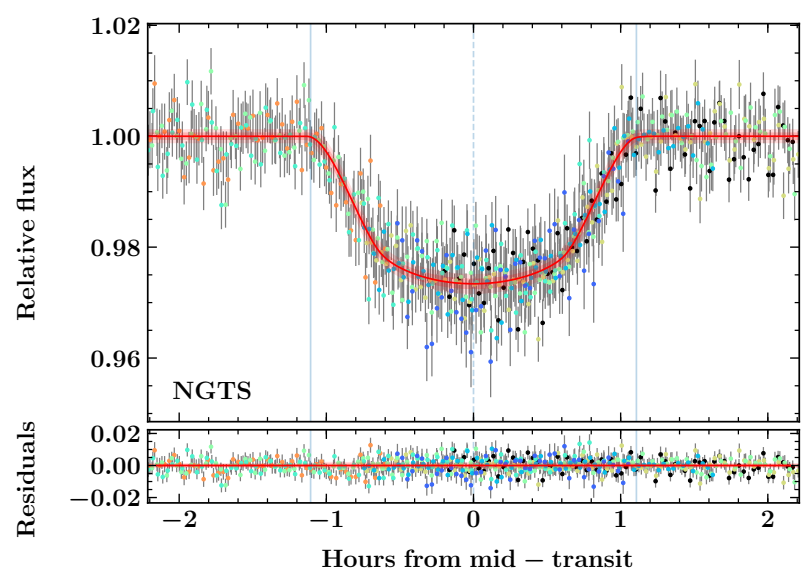

Fig. 1. Phase-folded light curve including NGTS data. The red line and pink shaded region show the median and $2 \sigma$ confidence intervals of the posterior GP-EBOP model (Gillen et al. 2017). Differently coloured points represent data from different transits.

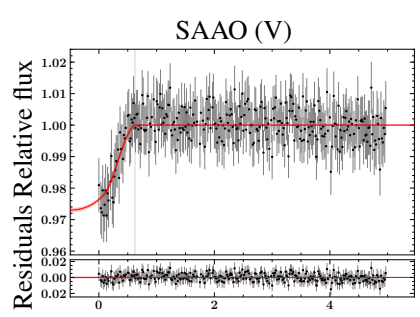

Euler (Z)

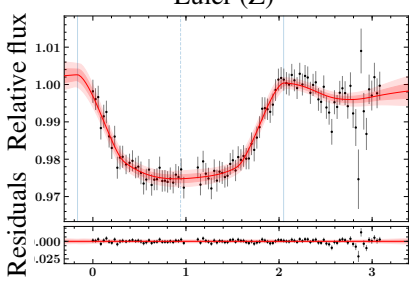

Time (hours)

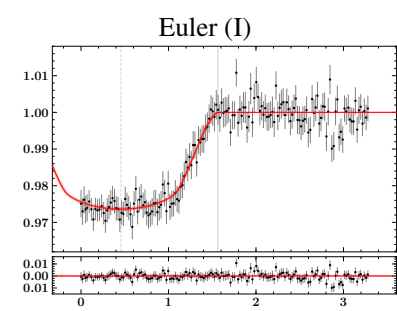

Euler (Z)

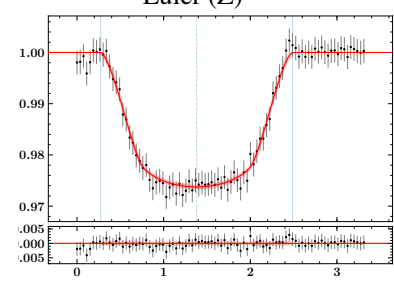

Fig. 2. Photometric follow-up light curves from SAAO (Sect. 2.2.1) taken in the night 2017 March 31 (top left) and Euler (Sect. 2.2.2). The Euler light curves were taken in the night 2017 July 13 (top right), 2017 July 23 (bottom left), and 2018 March 8 (bottom right). The red curve and the pink shaded regions show the best model fit and the 1 and $2 \sigma$ confidence intervals (same as described for Fig. 1).

\subsubsection{SAAO}

The transit of was observed through a Bessel $V$-band filter on the night of 2017 March 31 with the SHOC'n'awe camera, one of the frame-transfer CCD Sutherland High-speed Optical Cameras (Coppejans et al. 2013) mounted on the $1 \mathrm{~m}$ Elizabeth telescope at SAAO. The pixel scale of $0.167^{\prime \prime}$ pixel $^{-1}$ of these cameras is unnecessarily fine for our observations, therefore we binned $4 \times 4$ pixels in the $X$ and $Y$ directions. All observations were made in focus. A total of 900 frames with exposure times of $20 \mathrm{~s}$ were obtained over the course of the night. Calibration frames for the data reduction were taken at sunset and sunrise. To achieve a high precision, we combined the calibration frames with frames from the remaining observing run and performed bias correction and flat-fielding of the data. We used a background correction for the science frames, and differential photometry was performed using a 5.1 pixel aperture and three fainter comparison stars that are visible in the $2.85^{\prime} \times 2.85^{\prime}$ field of view. The comparison stars and aperture radius were chosen to maximise the signal-to-noise ratio $(\mathrm{S} / \mathrm{N})$ of the light curve.
All photometry was completed using the SEP python package (Barbary 2016), which is based on the core algorithms of the Source Extractor (Bertin \& Arnouts 1996).

\subsubsection{Euler}

We observed three transits of NGTS-5 with the Eulercam on the $1.2 \mathrm{~m}$ Euler Telescope at La Silla (Lendl et al. 2012) on the nights beginning 2017 July 13, 2017 July 23, and 2018 March 08. The first observation was made using a Cousins $I$ filter, a defocus of $0.15 \mathrm{~mm}$, and an exposure time of $60 \mathrm{~s}$. However, the first four exposures used an exposure time of $50 \mathrm{~s}$ and the following eight exposures used $70 \mathrm{~s}$ because the observations were fine-tuned to the target and conditions; there was some variable thin cloud during the night. The latter two observations used a Gunn $z$-filter and no defocus. The exposure time on 2017 July 23 was $90 \mathrm{~s}$ (with two exposures of $100 \mathrm{~s}$ and two exposures of $120 \mathrm{~s}$ for finetuning), while on 2017 July 23, we used $120 \mathrm{~s}$ for the duration. The gap of $\sim 2$ min in the light curve on 2017 July 23 is due to an error in the telescope control software.

In all cases, the data were reduced using the standard procedure of bias subtraction and flat-field correction. Aperture photometry was performed with the PyRAF implementation of the phot routine. PyRAF was also used to extract information that was useful for de-trending; $x$ - and $y$-position, FWHM, airmass, and sky background of the target star. The comparison stars and the photometric aperture radius were chosen in order to minimise the RMS scatter in the out-of-transit portion of the light curve.

\subsection{Spectroscopic follow-up}

Initial vetting of NGTS-5 was performed with the CORALIE spectrograph (Queloz et al. 2001b) on the Swiss $1.2 \mathrm{~m}$ telescope at La Silla Observatory, Chile. After checking for spectral binaries and blending scenarios, we obtained multi-epoch spectroscopy with the HARPS spectrograph (Mayor et al. 2003) on the ESO $3.6 \mathrm{~m}$ telescope at La Silla Observatory, Chile, between March 2017 and May 2018 under programs 098.C-0820(A), 099.C-0303(A), 0100.C-0474(A), and 0101.C-0623(A).

Radial velocities were calculated with a binary G2 mask using the standard data reduction pipelines for CORALIE and HARPS. The results are presented in Table 1. Initial analysis confirmed the planetary nature of the candidate, showing radial velocity variations in phase with the NGTS ephemerides with a semi-amplitude of $K \sim 40 \mathrm{~m} \mathrm{~s}^{-1}$. A single measurement was corrected for moon-light contamination using the simultaneous sky-fibre.

To ensure that the radial velocity signal does not originate from cool stellar spots or a blended eclipsing binary, we checked for correlations between the line bisector spans (Queloz et al. 2001a) and the radial velocity measurements. We find no evidence for a correlation; see Fig. 3. In order to characterise the stellar properties of NGTS-5, we combine and shifted the 17 HARPS spectra in wavelength that were not contaminated by moon light to create a spectrum with a high $\mathrm{S} / \mathrm{N}$ for the analysis in Sect. 3.1.

\section{Analysis}

\subsection{Stellar classification}

With data from the Gaia DR2, the stellar mass and radius can now be determined directly from the bolometric flux, effective 
Table 1. Coralie and HARPS radial velocity measurements of NGTS-5.

\begin{tabular}{lccr}
\hline \hline $\begin{array}{l}\text { BJD } \\
-2450\end{array}$ & $\begin{array}{c}\text { Radial velocity } \\
\left(\mathrm{km} \mathrm{s}^{-1}\right)\end{array}$ & $\begin{array}{c}\sigma_{\mathrm{RV}} \\
\left(\mathrm{km} \mathrm{s}^{-1}\right)\end{array}$ & $\begin{array}{r}\text { Instr. } \\
7626.507247\end{array}$ \\
7630.508626 & -5.593 & 0.051 & Coralie \\
7631.486303 & -5.552 & 0.074 & Coralie \\
7632.478367 & -5.605 & 0.044 & Coralie \\
7814.823955 & -5.5404 & 0.078 & Coralie \\
7924.610489 & -5.4903 & 0.0113 & HARPS \\
7925.621469 & -5.5545 & 0.0145 & HARPS \\
7960.533932 & -5.5245 & 0.0225 & HARPS \\
$7961.544233(a)$ & -5.5028 & 0.0519 & HARPS \\
7964.537489 & -5.5043 & 0.0331 & HARPS \\
7974.501742 & -5.4958 & 0.0106 & HARPS \\
7979.480682 & -5.5620 & 0.0114 & HARPS \\
7980.488371 & -5.5524 & 0.0145 & HARPS \\
7982.484781 & -5.5618 & 0.0117 & HARPS \\
7983.480932 & -5.5754 & 0.0121 & HARPS \\
8159.833208 & -5.4928 & 0.0140 & HARPS \\
8162.826420 & -5.4702 & 0.0101 & HARPS \\
8188.813394 & -5.4893 & 0.0087 & HARPS \\
8189.814538 & -5.4788 & 0.0071 & HARPS \\
8199.758555 & -5.4793 & 0.0069 & HARPS \\
8258.758482 & -5.5439 & 0.0088 & HARPS \\
8259.665546 & -5.4795 & 0.0094 & HARPS \\
\hline & & &
\end{tabular}

Notes. ${ }^{(a)}$ Corrected for moon-light contamination.

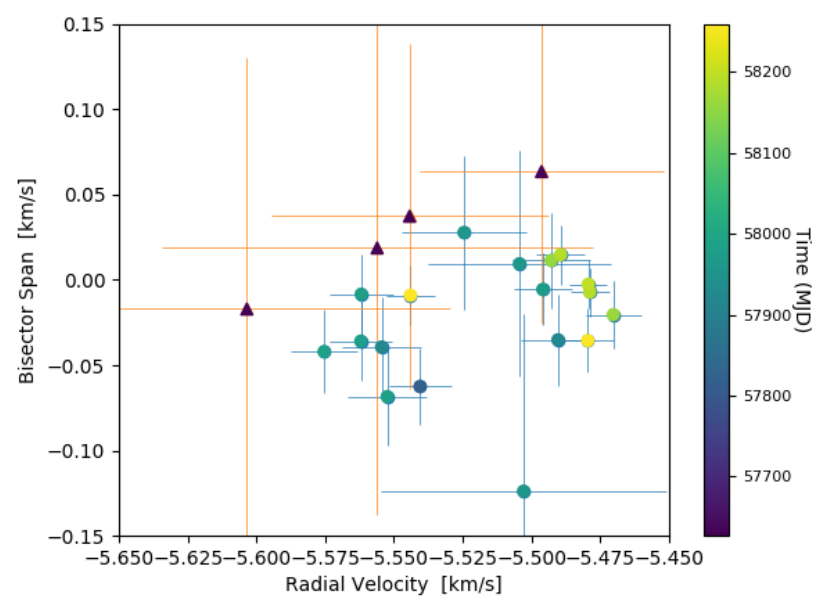

Fig. 3. Bisector span over radial velocity measurements with HARPS (blue error bars) and CORALIE (orange error bars). No correlation is visible.

temperature, and parallax. Applying the method described in Gillen et al. (2017), we used broadband photometric magnitudes from different catalogues as given in Table 2 (Henden et al. 2016; Abazajian et al. 2009; Skrutskie et al. 2006; Gaia Collaboration 2018; Wright et al. 2010; Kaiser et al. 2002) together with astrometric information from Gaia DR2 (Gaia Collaboration 2018) to determine the stellar parameters to high accuracy. The spectral energy distribution (SED) was modelled by convolving PHOENIX (Husser et al. 2013) and BT-SETTL (Allard et al. 2012) models using Markov chain Monte Carlo (MCMC) simulations. The best-fitting PHOENIX model is shown in Fig. 4.
Table 2. Main identifiers, coordinates, magnitudes, and spectroscopic parameters of NGTS-5.

\begin{tabular}{lcc}
\hline \hline Parameter & NGTS-5 & Unit \\
\hline RAJ2000 (Gaia DR1) & $14^{\mathrm{h}} 44^{\mathrm{m}} 13^{\mathrm{s}} .9842$ & $\mathrm{~h}$ \\
DECJ2000 (Gaia DR1) & $+05^{\circ} 36^{\prime} 19^{\prime \prime} .347$ & $\mathrm{deg}$ \\
Gaia DR2 source ID & 1159042833731813760 & $\ldots$ \\
Parallax & $3.2310 \pm 0.0272$ & $\mu \mathrm{as}$ \\
2MASS ID & $14441396+0536195$ & $\ldots$ \\
\hline Effective temperature $T_{\text {eff }}$ & $4987 \pm 41$ & $\mathrm{~K}$ \\
Surface gravity log $g_{*}$ & $4.52 \pm 0.037$ & $\mathrm{cgs}$ \\
Metallicity [Fe/H] & $0.12 \pm 0.1$ & $\mathrm{dex}$ \\
Spectral type & $\mathrm{K} 2 \mathrm{~V}$ & $\ldots$ \\
\hline Primary mass $M_{*}$ & $0.661_{-0.061}^{+0.068}$ & $M_{\odot}$ \\
Primary radius $R_{*}$ & $0.739_{-0.012}^{+0.014}$ & $R_{\odot}$ \\
\hline APASS $B$ & $14.706 \pm 0.052$ & $\mathrm{mag}$ \\
APASS $V$ & $13.770 \pm 0.054$ & $\mathrm{mag}$ \\
SDSS $g$ & $14.189 \pm 0.032$ & $\mathrm{mag}$ \\
SDSS $r$ & $13.483 \pm 0.031$ & $\mathrm{mag}$ \\
SDSS $i$ & $13.218 \pm 0.024$ & $\mathrm{mag}$ \\
PSg_AB & $14.132 \pm 0.003$ & $\mathrm{mag}$ \\
PSr_AB & $13.484 \pm 0.000$ & $\mathrm{mag}$ \\
PSz_AB & $13.173 \pm 0.010$ & $\mathrm{mag}$ \\
PSy_AB & $13.086 \pm 0.004$ & $\mathrm{mag}$ \\
Gaia G & $13.526 \pm 0.002$ & $\mathrm{mag}$ \\
Gaia BP & $14.024 \pm 0.002$ & $\mathrm{mag}$ \\
Gaia RP & $12.893 \pm 0.003$ & $\mathrm{mag}$ \\
2MASS J & $12.117 \pm 0.023$ & $\mathrm{mag}$ \\
2MASS H & $11.708 \pm 0.027$ & $\mathrm{mag}$ \\
2MASS Ks & $11.612 \pm 0.025$ & $\mathrm{mag}$ \\
WISE-1 & $11.526 \pm 0.023$ & $\mathrm{mag}$ \\
WISE-2 & $11.575 \pm 0.021$ & $\mathrm{mag}$ \\
\hline & & \\
& & \\
& &
\end{tabular}

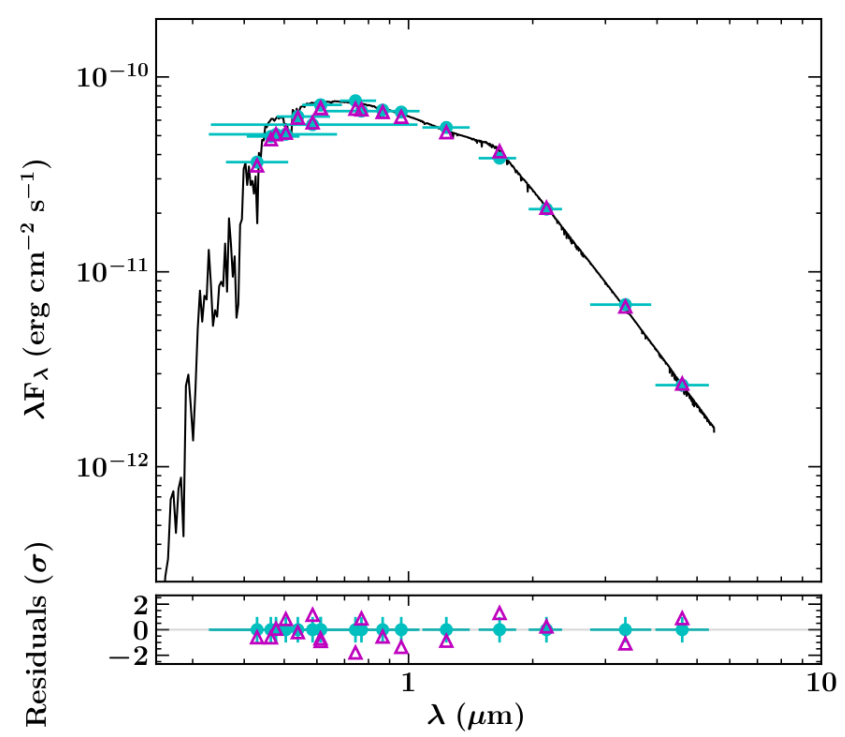

Fig. 4. Best-fitting PHOENIX model SED (black line) for NGTS-5 based on the photometric data (cyan points) presented in Table 2. The horizontal cyan lines indicate the widths of the different photometric bands. The magenta triangles represent the model flux convolved with each bandpass. Bottom: residuals of the fit in units of observational uncertainty. 
The resulting stellar parameters are in agreement with those that were determined using spectral classifications on the calibrated HARPS spectra, taken for radial velocity follow-up. For comparison we used a standard spectral classification following a method similar to the method reported by Doyle et al. (2013) and the Empirical SpecMatch tool as described in Yee et al. (2017). The results from all three methods agree with each other.

To obtain the most accurate stellar parameters, we determined the stellar mass by combining the very precise radius estimate from the above method with the stellar density as retrieved from the light curve analysis described in Sect. 3.2. The host star is a K2 dwarf with a radius of $R_{*}=0.739_{-0.012}^{+0.014} R_{\odot}$, a mass of $M_{*}=0.661_{-0.061}^{+0.068} M_{\odot}$, and an effective temperature of $T_{\text {eff }}=4987 \pm 41 \mathrm{~K}$. The catalogue information for the host star and the stellar parameters is listed in Table 2. We analysed the stellar spectra and photometric data for signs of stellar activity. The $\mathrm{S} / \mathrm{N}$ of the spectrum is too around the $\mathrm{Ca} \mathrm{H+K}$ lines to conclude about stellar activity. There are no emission lines above the noise. The $\mathrm{H}$-alpha profiles look normal, with no obvious signs of any emission or core infilling. The photometric data show no indications of stellar activity either.

\subsection{Combined modelling}

We modelled the light curves from NGTS and photometric follow-up observations together with the radial velocity data obtained at HARPS using GP-EBOP (Gillen et al. 2017). The radial velocity data showed a long-term trend in addition to the expected Keplerian orbit modulation. Furthermore, the CORALIE and HARPS data did not overlap in the timing of their observations; the CORALIE data were taken about six months before the HARPS observations. Because of the long-term trend, an unknown radial velocity offset between the zero-points of the two spectrographs, and large uncertainties on the CORALIE data relative to the semi-amplitude of the orbital modulation, we opted to remove the CORALIE data from our global modelling. Including them would have added model parameters and a correlation between the spectrograph zero-point offsets and the longterm trend with no obvious gain in radial velocity constraint. The main parameters we modelled were the sum of radii relative to the semi-major axis of system, the radius ratio, the cosine of the orbital inclination, the orbital period and epoch, the systemic radial velocity, the semi-amplitude of the radial velocity, and the sine of the linear radial velocity trend. For the limb darkening we assumed a quadratic law (using LDtk; Parviainen \& Aigrain 2015), which gave two additional free parameters for each photometric instrument (NGTS, SAAO, and EulerCam). The light curves with the best-fit model are shown in Figs. 1 and 2. We observe no transit timing variation of the transits.

We also modelled the system while allowing the eccentricity to vary. We found a best-fit eccentricity of $0.18 \pm 0.11$. An additional test, fitting the data allowing for eccentricity but not for a linear trend gave us an eccentricity of $0.11 \pm 0.08$. Because the observations are limited in number, we found that the bestfit eccentricity was not significant and therefore decided for the final model to fix the system on a circular orbit. The implications for the radius and mass of the planetary companion were negligible in either case.

The residuals in the fit to the radial velocity data suggest that the assumed linear trend does not describe the observational data well. Fitting a second Keplerian solution to the residuals would be over-fitting the available data, however. The best-fit model together with the radial velocity data is shown in Fig. 5 (phase folded) and Fig. 6 (over time).

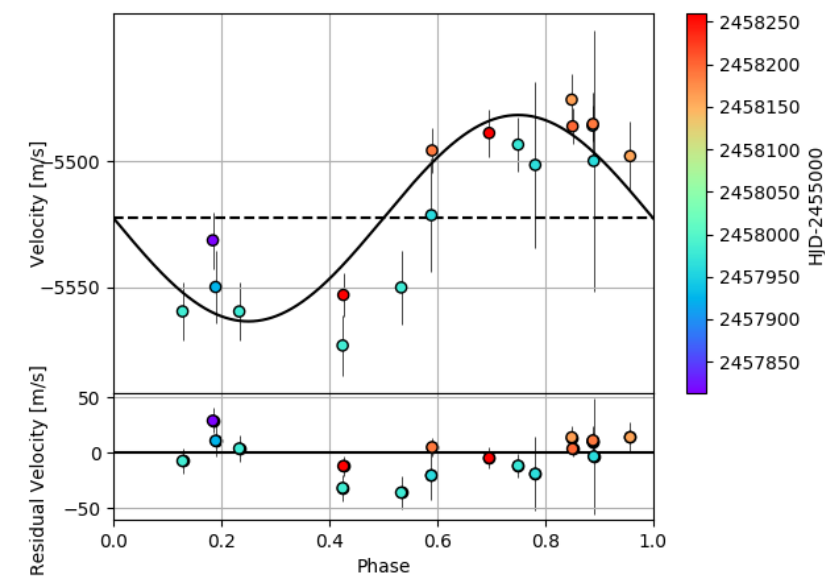

Fig. 5. Modelled radial velocity measurements in orbital phase, colourcoded by the time of observation. The fit of the best orbital solution is given by the black line.

The MCMC posterior distribution for the main parameters is shown in Fig. 7.

\section{Results}

The K2 main-sequence star NGTS-5 is orbited by shortperiod exoplanet. NGTS-5b transits its star every $3.3569866 \pm$ 0.0000026 days. The orbit is slightly inclined with an inclination of $i=86.6 \pm 0.2^{\circ}$. The eccentricity is not well constrained from our radial velocity follow-up observations. We therefore assumed the orbit to be circular. The residuals in the radial velocity data might be due to an additional orbiting element in the system, but the data at hand do not allow us to restrict its parameters. For our final modelling we applied a linear trend to account for the additional signal. The residuals in Fig. 6 show that a linear trend might not be the best explanation for the additional signal, but more free parameters would lead to over-fitting the data. To understand the implications to the bulk properties of the planet, we modelled the system with eccentricity as a free parameter, as well as without any additional trend and assuming a quadratic trend. The bulk parameters of the planet did not change significantly and were consistent within $1 \sigma$.

The transit light curves allow us to constrain that the radius of the planet is slightly larger than the radius of Jupiter with $R_{\mathrm{P}}=1.136 \pm 0.023 R_{\text {Jup }}$. The radial velocity follow-up measurements, however, show the mass to be significantly lower than the mass of Jupiter, $M_{\mathrm{P}}=0.229 \pm 0.037 M_{\text {Jup }}$, resulting in an extremely low mean density of $0.19 \mathrm{~g} \mathrm{~cm}^{-3}$.

\section{Discussion}

With its short orbital period and low mass, NGTS-5b lies within the mass/period sub-Jovian desert as defined in Mazeh et al. (2016). In Fig. 8, NGTS-5b is plotted (blue) together with other planets selected from the NASA Exoplanet Archive ${ }^{1}$ (Akeson et al. 2013) (coloured points highlight planets which are used later when discussing Figs. 9-11). The black dashed lines correspond to the empirically defined boundaries of the sub-Jovian desert (Mazeh et al. 2016). The background and the dotted line represent the point density present in our planet sample. The

1 The data we used include all confirmed planets with an uncertainty in radius lower than $10 \%$ and an uncertainty in mass lower than $50 \%$ as available on 18 April 2019. 


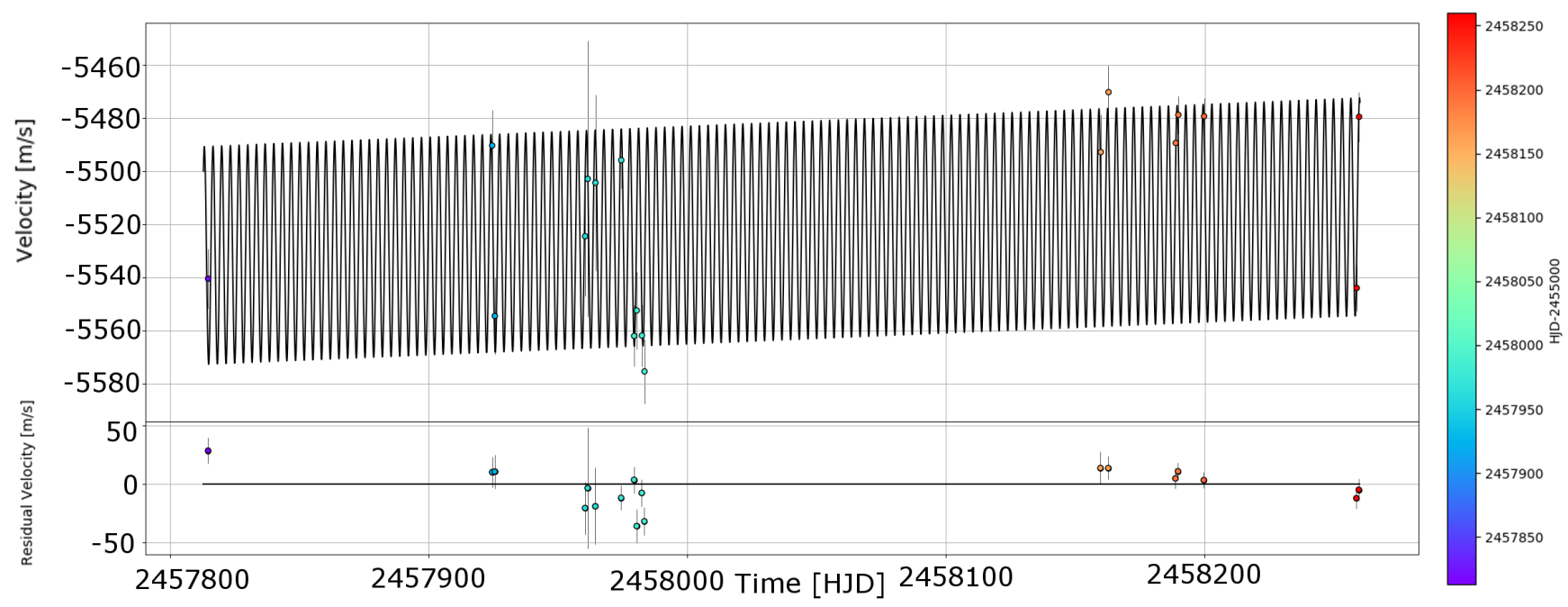

Fig. 6. Radial velocity measurements over time, colour-coded by the time of observation. The fit of the best orbital solution including a linear trend is given by the black line.

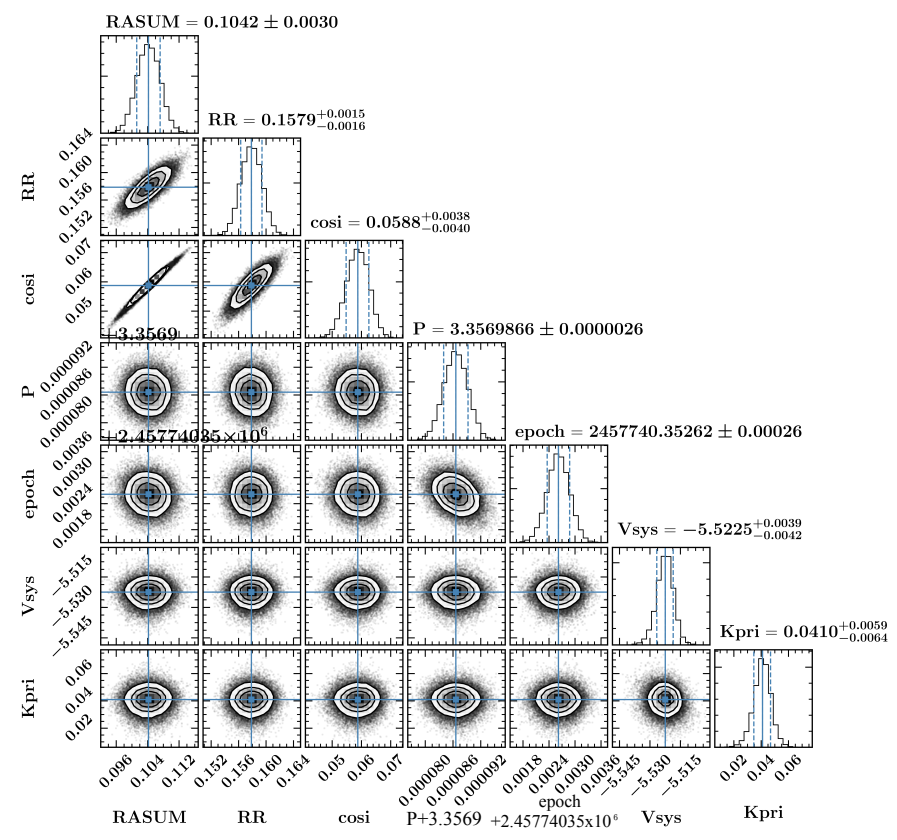

Fig. 7. MCMC posterior distribution for the main free parameters.

magenta region in Fig. 8 represents the high-eccentricity migration boundary including tidal decay as presented in Owen \& Lai (2018). In comparison with the boundary from Mazeh et al. (2016) NGTS-5b lays within the desert, whereas the theoretical boundary as well as the point density of current exoplanet population suggest NGTS-5b to be closer to the upper boundary of the sub-Jovian desert.

In the radius-period plane (Fig. 10) NGTS-5b is well above the boundary presented by Mazeh et al. (2016) and lies in the middle of the population of inflated hot Jupiters.

Figures 8 and 10 display the sub-Jovian desert as it is typically shown (e.g. Mazeh et al. 2016; Matsakos \& Königl 2016; Owen \& Lai 2018). This however does not take the stellar information of the planet host into account, but instead assumes all host stars to be solar like. As NGTS-5 cannot be approximated with solar parameters, our conception of NGTS-5b in regards to the sub-Jovian desert, as depicted in Figs. 8 and 10 may be misleading. A recent study by Szabó \& Kálmán (2019), which was published during our reviewing process, also shows the dependence of the sub-Jovian desert on stellar parameters. Processes thought to be responsible for shaping the sub-Jovian desert are related to the host stars' parameters. One effect which may be responsible for shaping the upper boundary of the sub-Jovian desert is inflation caused by e.g. ohmic heating. This effect is not directly correlated to the orbital period. The direct dependence of inflation would be to the insolation or equilibrium temperature of the planet. By using the planet's equilibrium temperature instead of the orbital period, we thus show the dependency caused by inflation when the host star is taken into account. Figure 11 can be directly compared to Fig. 10 . The $y$-axis is the same, but for the $x$-axis, the orbital period has been replaced by the planetary equilibrium temperature assuming an albedo of 0.2 for all planets. For NGTS-5b, K2-60b, NGTS-1b, NGTS-2b, and NGTS-4b, the difference between assuming solar properties for the host star (shaded) and using the actual host star parameters is highlighted with arrows. The properties of these planets are listed in Table 4. This difference directly shows the importance of taking stellar properties into account depending on the stellar type. K2-60 is a G-type star, therefore changes are not significant. NGTS-5b, NGTS-1b, and NGTS-4b all orbit K- or M-dwarfs and show a clear shift to lower equilibrium temperatures. NGTS-2, on the other hand, is an F-type star, which is the reason that we can see the opposite effect. In addition to the significant influence on single planets, such as NGTS-5b, the shape of the hot-Jupiter population is also affected in general. A comparison of Figs. 10 and 11 shows that the equilibrium temperature is more strongly dependent on the radius at the upper boundary of the sub-Jovian desert. The slope of the contour lines below the population of the hot Jupiters, which is indicative of the upper boundary of the sub-Jovian desert, also changes and agrees better with the results of Mazeh et al. (2016) when stellar type is accounted for (cf. Fig. 11). This shows how much more diverse our sample is than the sample that was analysed in Mazeh et al. (2016).

Next to the inflation, which is related to the stellar insolation, another effect that shapes the desert is high-eccentricity migration. As a dynamical process, it is not directly dependent on the stellar insolation, but is instead directly correlated with the orbital period of the planet. However, the host star also plays 
Table 3. Parameters from light curve and radial velocity data analysis.

\begin{tabular}{|c|c|c|}
\hline Parameter & Combined modelling & Unit \\
\hline Orbital period $P_{\text {orb }}$ & $3.3569866 \pm 0.0000026$ & days \\
\hline Transit epoch $T_{0}$ & $7740.35262 \pm 0.00026$ & $\mathrm{BJD}_{\mathrm{TDB}}-2450000$ \\
\hline Transit duration & $2.21 \pm 0.03$ & $\mathrm{~h}$ \\
\hline Scaled semi-major axis $a / R_{*}$ & $11.111_{-0.296}^{+0.315}$ & \\
\hline Semi-major axis $a$ & $0.0382 \pm 0.0013$ & $\mathrm{au}$ \\
\hline Scaled secondary radius $R_{2} / R_{1}$ & $0.1579 \pm 0.0016$ & \\
\hline Orbital inclination angle $i$ & $86.6 \pm 0.2$ & deg \\
\hline Limb-darkening coefficient $\mathrm{z} u_{+}$ & $0.29 \pm 0.02$ & \\
\hline Limb-darkening coefficient $\mathrm{z} u_{-}$ & $0.40 \pm 0.02$ & \\
\hline Limb-darkening coefficient $\mathrm{I} u_{+}$ & $0.35 \pm 0.03$ & \\
\hline Limb-darkening coefficient $\mathrm{I} u_{-}$ & $0.41 \pm 0.02$ & \\
\hline Limb-darkening coefficient $\mathrm{V} u_{+}$ & $0.59 \pm 0.04$ & \\
\hline Limb-darkening coefficient $\mathrm{V} u_{-}$ & $0.48 \pm 0.02$ & \\
\hline Limb-darkening coefficient NGTS $u_{+}$ & $0.44 \pm 0.03$ & \\
\hline Limb-darkening coefficient NGTS $u_{-}$ & $0.44 \pm 0.02$ & \\
\hline Radial velocity semi-amplitude $K$ & $41.0_{-6.4}^{+5.9}$ & $\mathrm{~m} \mathrm{~s}^{-1}$ \\
\hline Systemic radial velocity $\gamma$ & $-5.5225_{-0.0042}^{+0.0039}$ & $\mathrm{~km} \mathrm{~s}^{-1}$ \\
\hline Sine of the linear radial velocity trend & $0.015 \pm 0.012$ & $\left(\mathrm{~km} \mathrm{~s}^{-1} \mathrm{yr}^{-1}\right)$ \\
\hline Eccentricity $e$ & 0.0 (fixed) & \\
\hline Secondary mass $M_{\mathrm{P}}$ & $0.229 \pm 0.037$ & $M_{\text {Jup }}$ \\
\hline Secondary radius $R_{\mathrm{P}}$ & $1.136 \pm 0.023$ & $R_{\text {Jup }}$ \\
\hline Secondary surface gravity $\log g_{\mathrm{p}}$ & $2.643_{-0.078}^{+0.066}$ & $\operatorname{cgs}$ \\
\hline Secondary calculated effective temperature & $952 \pm 24$ & $\mathrm{~K}$ \\
\hline
\end{tabular}

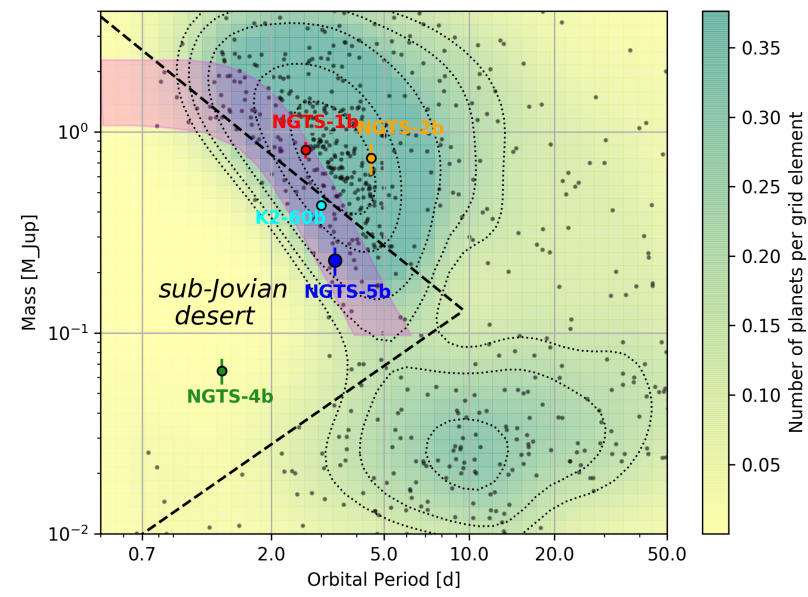

Fig. 8. Planetary mass over orbital period. Plotted are all stars from the NASA Exoplanet Archive with a radius uncertainty lower than $10 \%$ or a mass uncertainty lower than $50 \%$. The blue point shows NGTS-5b. Additionally, we highlight NGTS-1b in red (Bayliss et al. 2018), NGTS-2b in orange (Raynard et al. 2018), NGTS-4b in green (West et al. 2019), and K2-60b in cyan (Eigmüller et al. 2017). The black dashed lines show the boundaries of the sub-Jovian desert as determined in Mazeh et al. (2016). The magenta region shows the high-eccentricity migration boundary including tidal decay as presented in Owen \& Lai (2018). The background and the dotted black lines highlight the point density per grid element (the plot is divided into $40 \times 40$ equally spaced grid elements) of our sample. The yellow region on the left shows the sub-Jovian desert.

a crucial role in this effect because the dynamical processes are correlated to the stellar mass. Matsakos \& Königl (2016)

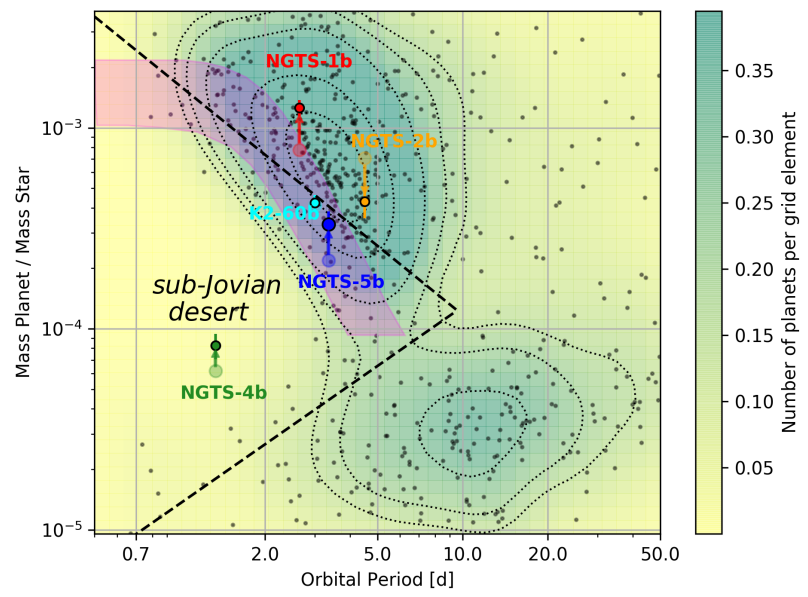

Fig. 9. Mass ratio (planetary mass over stellar mass) plotted over the orbital period. The data are the same as in Fig. 8. The black dashed lines and magenta region show the boundaries of the sub-Jovian desert as in Fig. 8, but converted into the mass ratio assuming solar mass for the host star. Highlighted are NGTS-5b (blue, K-dwarf host), NGTS-1b (red, M-dwarf host), NGTS-2b (orange, F-dwarf host), NGTS-4b (green, K-dwarf host), and K2-60b (cyan, G-dwarf host). To highlight the influence of the stellar type, we also show for these stars the position in the diagram; the sun is also assumed to be a host star (i.e. stellar parameters are not taken into account; shaded). The arrows show the change that is effected when the host star is taken into account.

modelled only planets orbiting solar mass stars, which required them to take the host star into account when they displayed the sub-Jovian desert. Equations (3) and (4) in Matsakos \& Königl (2016) clearly show, however, that the mass of the host star 


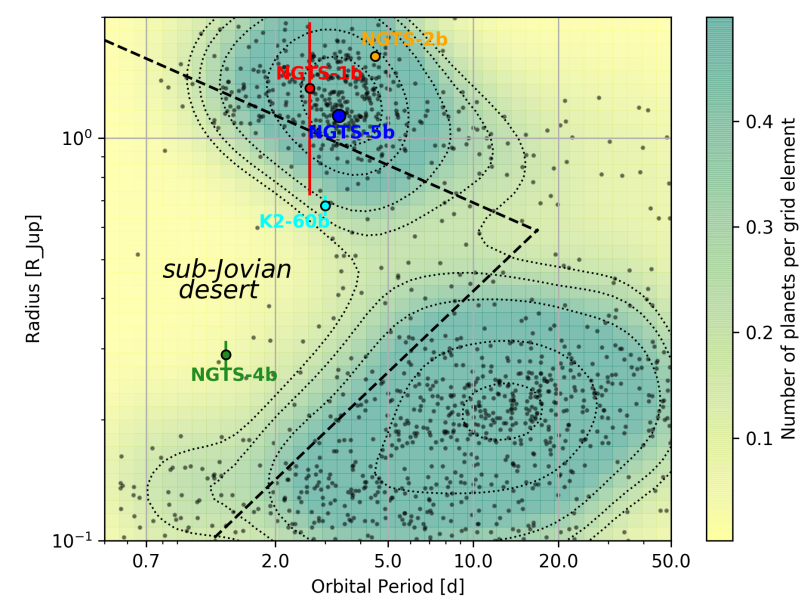

Fig. 10. Same as Fig. 8, but for the planetary radius over orbital period.

should be taken into account in a more diverse sample. In Fig. 9 we show the sub-Jovian desert by plotting the mass ratio over the orbital period. As in Fig. 11, we highlight the planets NGTS-5b, K2-60b, NGTS-1b, NGTS-2b, and NGTS-4b and the influence of their host star on their position in this diagram in relation to the overall planet population.

When the host star is taken into account, NGTS-5b is shifted from within the desert to its upper boundary. NGTS-4b moves more into the middle of the desert, but NGTS-4b with its low mass might belong to a different planet population than NGTS-5b and thus may be affected by additional effects such as photo-evaporation. In contrast to Fig. 11, Fig. 9 does not show a significant change in the shape of the planetary population of hot Jupiters when the stellar parameters are taken into account.

Together with inflation and high-eccentricity migration, photo-evaporation might also play a role in shaping the subJovian desert, especially at the lower boundary. Photoevaporation is caused by the EUV or X-ray radiation of its hosts star. The planet is mainly exposed to this radiation in its early evolutionary stages, and for most planets, we have no knowledge of the amount of EUV or X-ray radiation that the planet received. As a first estimate, we suggest that the equilibrium temperature is a better approximation for the amount of EUV or X-ray heating or radiation than the orbital period.

In addition to its position in regard to the sub-Jovian desert, NGTS-5b is also interesting because of its unusually low density. In Fig. 12 we show the density of planets that have been reported in the literature as a function of the equilibrium temperature. NGTS-5b is at the lower limit of what has been found so far in its temperature regime. The lower boundary of the planetary population in this parameter space might be directly related to inflation. NGTS-5b lies directly at the lower limit of observed densities in the temperature regime around $T_{\text {eq }} \approx 1000 \mathrm{~K}$. The contour lines for the planet population point density show a sharp boundary for the lowest possible densities depending on the equilibrium temperature.

\section{Conclusions}

We reported the discovery of NGTS-5b with a mass of $0.229 \pm$ $0.037 M_{\text {Jup }}$ and radius of $1.136 \pm 0.023 R_{\text {Jup }}$. Its low mass places NGTS-5b at the edge of the sub-Jovian desert. NGTS-5 is expected to be observed by TESS in sector 24. The TESS sector 24 observations are scheduled to start in April 2020 and continue into May 2020.

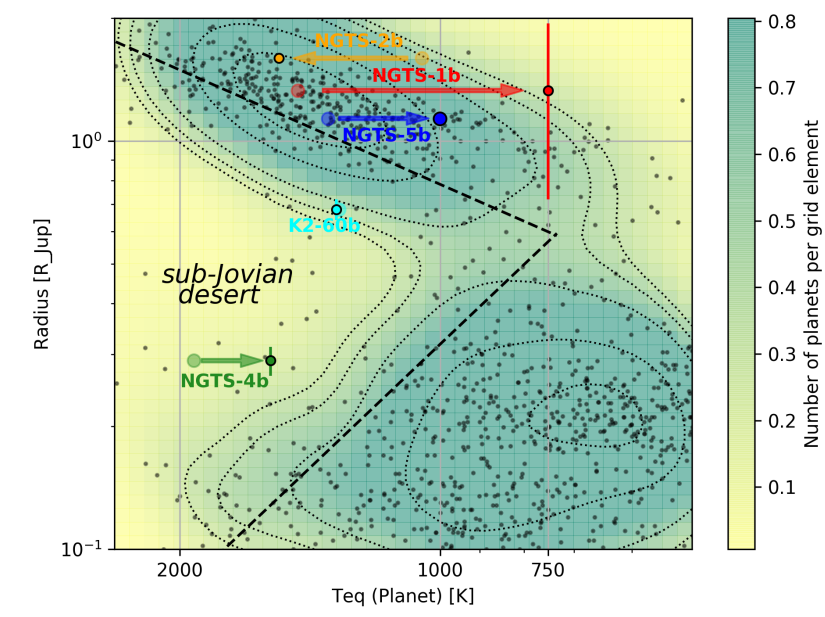

Fig. 11. Same as Fig. 9, but for the planetary radius over equilibrium temperature.

Table 4. Parameters for the selected planets used in Figs. 8-11.

\begin{tabular}{clcccccc}
\hline \hline Planet & $\begin{array}{l}R_{\mathrm{P}} \\
\left(R_{\text {Jup }}\right)\end{array}$ & $\begin{array}{c}M_{\mathrm{P}} \\
\left(M_{\text {Jup }}\right)\end{array}$ & $\begin{array}{c}\mathrm{P} \\
(\mathrm{d})\end{array}$ & $\begin{array}{c}T_{\text {eq }} \\
(\mathrm{K})\end{array}$ & $\begin{array}{c}R_{*} \\
\left(R_{\odot}\right)\end{array}$ & $\begin{array}{c}M_{*} \\
\left(M_{\odot}\right)\end{array}$ & $\begin{array}{c}T_{\text {eff }} \\
(\mathrm{K})\end{array}$ \\
\hline NGTS-1b & $1.33^{(a)}$ & 0.81 & 2.65 & 750 & 0.57 & 0.62 & 3916 \\
NGTS-2b & 1.60 & 0.74 & 4.51 & 1535 & 1.70 & 1.64 & 6478 \\
NGTS-4b & 0.29 & 0.06 & 1.33 & 1572 & 0.84 & 0.75 & 5143 \\
NGTS-5b & 1.14 & 0.23 & 3.36 & 1001 & 0.74 & 0.66 & 4987 \\
K2-60b & 0.68 & 0.43 & 3.00 & 1318 & 1.12 & 0.97 & 5500 \\
\hline
\end{tabular}

Notes. The table lists: planetary radius, planetary mass, orbital period, equilibrium temperature, stellar radius, stellar mass, and stellar effective temperature. ${ }^{(a)}$ NGTS-1b only shows grazing transits. The radius of the planet therefore has large uncertainties.

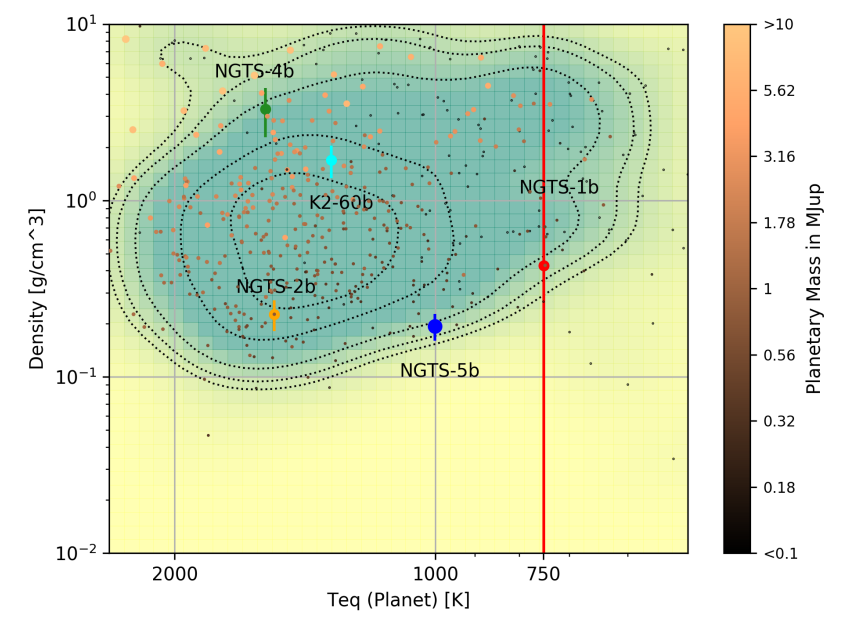

Fig. 12. Density over equilibrium temperature colour-coded by planetary mass. Data and background are the same as above.

A comparison of the upper boundary of the sub-Jovian desert as defined by the point density contour lines in Figs. 9 and 11 to the sub-Jovian desert as empirically determined by Mazeh et al. (2016) reveals discrepancies in the slope. The theoretical upper boundary that is due to high-eccentricity migration as given in Owen \& Lai (2018) agrees better with the observed point density.

To allow us to account for the stellar type when we set this planet in context with the known planet population, we 
introduced a presentation of the sub-Jovian desert in a parameter space that includes the influence of the host star in the planetary evolution. Using the mass ratio between the planet and host star instead of the planetary mass, we accounted for the stellar mass that affects the high-eccentricity migration. To take the luminosity of the host star into account when we studied the inflation of hot Jupiters, we considered the planetary radius as a function of equilibrium temperature instead of the orbital period.

Taking the host star and its mass and luminosity into account changed the location of NGTS-5b and other planets especially around low-mass stars in regard to the sub-Jovian desert. NGTS-5b is now at the boundary of the sub-Jovian desert in regard to its mass, when otherwise it would lie more in the centre of the desert. Judging from its radius, it is clearly outside the desert and at the upper edge of a population of inflated planets. Together with the low mass, this leads to a very low density for this equilibrium temperature. The density as a function of equilibrium temperature places NGTS-5bat the lower edge of observed densities. The observed significant changes in the position of single objects in regard to the sub-Jovian desert, or more generally, in regard to planet populations depending on the stellar type of the host star, highlights the care that is required when planet populations are to be discussed. We showed that in case of the sub-Jovian desert, a population analysis that does not take the host star into account might lead to incorrect findings. In order to simplify a concept such as the sub-Jovian desert, which might depend on several parameters, into a 2D plot, the parameter space needs to be selected carefully. We suggest that the sub-Jovian desert might not be displayed not by planetary mass or radius over orbital period, as shown in Figs. 8 and 10, but by taking the stellar type into account, as shown in Figs. 9 and 11.

Our presentation of the sub-Jovian desert, which includes information about the host star, will allow us to directly compare observed planet populations of a inhomogeneous sample with model predictions. With red-sensitive planet surveys such as TESS and NGTS, we will continue to detect planets around late-type stars. For these planets it is important to take the host star into account when the planet population in general and the sub-Jovian desert specifically are studied.

Acknowledgements. This work is based on data collected under the NGTS project at the ESO La Silla Paranal Observatory. The NGTS facility is operated by the consortium institutes with support from the UK Science and Technology Facilities Council (STFC) project ST/M001962/1. This paper uses observations made at the South African Astronomical Observatory (SAAO). The contributions at the University of Warwick by P.J.W., R.G.W., D.L.P., D.J.A., B.T.G. and T.L. have been supported by STFC through consolidated grants ST/L000733/1 and ST/P000495/1. Contributions at the University of Geneva by D.B., F.B., B.C., L.M., and S.U. were carried out within the framework of the National Centre for Competence in Research "PlanetS" supported by the Swiss National Science Foundation (SNSF). The contributions at the University of Leicester by M.R.G. and M.R.B. have been supported by STFC through consolidated grant ST/N000757/1. C.A.W. acknowledges support from the STFC grant ST/P000312/1. E.G. gratefully acknowledges support from Winton Philanthropies in the form of a Winton Exoplanet Fellowship. M.N.G. is supported by the STFC award reference 1490409 as well as the Isaac Newton Studentship. J.S.J. acknowledges support by Fondecyt grant 1161218 and partial support by CATA-Basal (PB06, CONICYT). D.J.A. gratefully acknowledges support from the STFC via an Ernest Rutherford Fellowship (ST/R00384X/1). P.E., A.C., and H.R. acknowledge the support of the DFG priority program SPP 1992 "Exploring the Diversity of Extrasolar Planets" (RA 714/13-1). L.D. acknowledges support from the Gruber Foundation Fellowship. The research leading to these results has received funding from the European Research Council under the FP/2007-2013
ERC Grant Agreement number 336480 and from the ARC grant for Concerted Research Actions, financed by the Wallonia-Brussels Federation. This work was also partially supported by a grant from the Simons Foundation (PI. Queloz, ID 327127). This work has made use of data from the European Space Agency (ESA) mission Gaia (https://www.cosmos.esa.int/gaia), processed by the Gaia Data Processing and Analysis Consortium (DPAC, https://www. cosmos.esa.int/web/gaia/dpac/consortium). Funding for the DPAC has been provided by national institutions, in particular the institutions participating in the Gaia Multilateral Agreement. PyRAF is a product of the Space Telescope Science Institute, which is operated by AURA for NASA. This research has made use of the NASA Exoplanet Archive, which is operated by the California Institute of Technology, under contract with the National Aeronautics and Space Administration under the Exoplanet Exploration Program.

\section{References}

Abazajian, K. N., Adelman-McCarthy, J. K., Agüeros, M. A., et al. 2009, ApJS, 182,543

Akeson, R. L., Chen, X., Ciardi, D., et al. 2013, PASP, 125, 989

Allard, F., Homeier, D., \& Freytag, B. 2012, Phil. Trans. R. Soc. London, Ser. A, 370,2765

Barbary, K. 2016, J. Open Source Softw., 1, 58

Bayliss, D., Gillen, E., Eigmüller, P., et al. 2018, MNRAS, 475, 4467

Bertin, E., \& Arnouts, S. 1996, A\&AS, 117, 393

Bodenheimer, P., Lin, D. N. C., \& Mardling, R. A. 2001, ApJ, 548, 466

Burrows, A., Hubeny, I., Budaj, J., \& Hubbard, W. B. 2007, ApJ, 661, 502

Chazelas, B., Pollacco, D., Queloz, D., et al. 2012, in Ground-based and Airborne Telescopes IV, Proc. SPIE, 8444, 84440E

Coppejans, R., Gulbis, A. A. S., Kotze, M. M., et al. 2013, PASP, 125, 976

Doyle, A. P., Smalley, B., Maxted, P. F. L., et al. 2013, MNRAS, 428, 3164

Eigmüller, P., Gandolfi, D., Persson, C. M., et al. 2017, AJ, 153, 130

Gaia Collaboration (Brown, A. G. A., et al.) 2018, A\&A, 616, A1

Gillen, E., Hillenbrand, L. A., David, T. J., et al. 2017, ApJ, 849, 11

Guillot, T., \& Showman, A. P. 2002, A\&A, 385, 156

Günther, M. N., Queloz, D., Gillen, E., et al. 2017, MNRAS, 472, 295

Günther, M. N., Queloz, D., Gillen, E., et al. 2018, MNRAS, 478, 4720

Henden, A. A., Templeton, M., Terrell, D., et al. 2016, VizieR Online Data Catalog: II/336

Howell, S. B., Sobeck, C., Haas, M., et al. 2014, PASP, 126, 398

Husser, T. O., Wende-von Berg, S., Dreizler, S., et al. 2013, A\&A, 553, A6

Irwin, M. J., Lewis, J., Hodgkin, S., et al. 2004, in Optimizing Scientific Return for Astronomy through Information Technologies, eds. P. J. Quinn \& A. Bridger, Proc. SPIE, 5493, 411

Jackman, J. A. G., Wheatley, P. J., Pugh, C. E., et al. 2018, MNRAS, 477, 4655

Kaiser, N., Aussel, H., Burke, B. E., et al. 2002, in Survey and Other Telescope Technologies and Discoveries, eds. J. A. Tyson \& S. Wolff, SPIE Conf. Ser., 4836, 154

Kovács, G., Zucker, S., \& Mazeh, T. 2002, A\&A, 391, 369

Laughlin, G., Crismani, M., \& Adams, F. C. 2011, ApJ, 729, L7

Lendl, M., Anderson, D. R., Collier-Cameron, A., et al. 2012, A\&A, 544, A72

Lundkvist, M. S., Kjeldsen, H., Albrecht, S., et al. 2016, Nat. Commun., 7, 11201

Matsakos, T., \& Königl, A. 2016, ApJ, 820, L8

Mayor, M., Pepe, F., Queloz, D., et al. 2003, The Messenger, 114, 20

Mazeh, T., Holczer, T., \& Faigler, S. 2016, A\&A, 589, A75

Owen, J. E., \& Lai, D. 2018, MNRAS, 479, 5012

Parviainen, H., \& Aigrain, S. 2015, MNRAS, 453, 3821

Queloz, D., Henry, G. W., Sivan, J. P., et al. 2001a, A\&A, 379, 279

Queloz, D., Mayor, M., Udry, S., et al. 2001b, The Messenger, 105, 1

Raynard, L., Goad, M. R., Gillen, E., et al. 2018, MNRAS, 481, 4960

Ricker, G. R., Latham, D. W., Vanderspek, R. K., et al. 2010, in Amer. Astron.

Soc. Meet. Abstr., 215, 450.06

Sestovic, M., Demory, B.-O., \& Queloz, D. 2018, A\&A, 616, A76

Skrutskie, M. F., Cutri, R. M., Stiening, R., et al. 2006, AJ, 131, 1163

Szabó, G. M., \& Kálmán, S. 2019, MNRAS, 485, L116

Tamuz, O., Mazeh, T., \& Zucker, S. 2005, MNRAS, 356, 1466

West, R. G., Gillen, E., Bayliss, D., et al. 2019, MNRAS, submitted [arXiv:1809.00678]

Wheatley, P. J., West, R. G., Goad, M. R., et al. 2018, MNRAS, 475, 4476

Wright, E. L., Eisenhardt, P. R. M., Mainzer, A. K., et al. 2010, AJ, 140, 1868

Yee, S. W., Petigura, E. A., \& von Braun, K. 2017, ApJ, 836, 77 